\title{
Synthesis of Highly Porous $\mathrm{SrTiO}_{3}$ Materials
}

\author{
A. Mizera, E. DrożDŻ AND Ł. ŁAŃCUCKI* \\ AGH University of Science and Technology, Faculty of Materials Science and Ceramics,
} al. Mickiewicza 30, 30-059 Kraków, Poland

\begin{abstract}
Strontium titanate is a perovskite with a great potential to act as an anode material for solid oxide fuel cells. The requirements for these materials concern not only electrical but also microstructural properties. The strontium titanate with regular pore structure can exhibit lower flow resistance compared to random pore distributed materials. Several methods of synthesis (with addition of various organic agents) allowed obtaining materials with regular/irregular pore structure. Regardless of synthesis methods all materials showed high porosity (above 40 vol.\%). Additionally it was found that three-dimensionally ordered macroporous structure collapsed after sintering at $1200{ }^{\circ} \mathrm{C}$ whereas macro-mesoporous structure of P-123 modified strontium titanate had persisted.
\end{abstract}

DOI: 10.12693/APhysPolA.133.873

PACS/topics: $81.20 .-\mathrm{n}, 61.10 . \mathrm{Nz}$

\section{Introduction}

Strontium titanate based perovskites are materials that can potentially be applied (after doping) as an alternative to $\mathrm{Ni} / \mathrm{YSZ}$ as anode backbone for solid oxide fuel cells (SOFCs). Apart from proper catalytic and oxygen conductive properties, obtained materials should fulfill other crucial requirements associated with their microstructure. It was found that the length of the three phase boundary $\mathrm{TPB}$ - created by electron-conductive, ion-conductive material and fuel (in the simplest case hydrogen) - should be as long as possible to ensure high efficiency of the electrode reaction. It was estimated that open porosity level should be greater than 30 vol.\% in order to be entirely serviceable as anode material in SOFC $[1,2]$. The use of regular pore structure materials can increase the fuel flow rate of the anode material relative to materials with random pore structure. A route of synthesis have a great impact on microstructural properties such as size, shape and distribution of pores. To increase level of porosity the classical solid-state synthesis can be replaced by one of wet preparation methods. The high porosity strontium titanate (STO) can be obtained by various modifications of sol-gel synthesis route. The synthesis of such porous perovskites involves several crucial steps that are: preparation of stable sol-gel initial solution, micelle formation by addition of various nonionic surfactants, deposition of solution on soft templates. Each of these steps is important since the configuration of the final matrix and its structure depend on the combination of inorganic precursor and surfactant porogen in the solution. Furthermore it depends on the conditions applied during elimination of the soft polystyrene templates and the stabilization of the inorganic network [3]. In this manuscript a focus on impact of various surfactants and soft template on STO microstructure was given.

\footnotetext{
*corresponding author; e-mail: lancucki@agh.edu.pl
}

\section{Experimental}

\subsection{Synthesis of perovskite and preparation of samples}

All $\mathrm{SrTiO}_{3}$ samples were prepared using citrate method. Firstly the required amount of citric acid was placed into flask containing ethanol solution of titanium(IV) isopropoxide. The mixture was stirred. After several minutes the aqueous solution of strontium nitrate was added. The obtained mixture was stirred for 15 min. The final concentration of all inorganic ions ( $\mathrm{Sr}, \mathrm{Ti})$ to citric acid was 1:2. Various organic compound such as Triton X-100, Pluronic P-125, poly (vinyl alcohol) (all in aqueous solutions) and polystyrene nanospheres were used. In the case of synthesis with polymeric soft template, the polystyrene (PS) spheres were immersed in precursor mixture, vacuum filtered, dried and calcinated. The calcination temperature for all samples was set at $900^{\circ} \mathrm{C}$ with a temperature ramp of $1^{\circ} \mathrm{C} / \mathrm{min}$. All samples were formed into pellets pressed at $2 \mathrm{MPa}$ and sintered at $1200^{\circ} \mathrm{C}$.

\subsection{Methods}

Phase compositions of the samples (calcined powders) were identified by X-ray diffraction analysis, based on the ICDD databases. XRD analysis was performed using Philips X'Pert Pro diffractometer.

The microstructure of all $\mathrm{SrTiO}_{3}$ prepared samples was examined by a scanning electron microscope (NOVA NANO SEM 200,FEI). The total porosity of samples was determined on the basis of geometric measurements and mass.

\section{Results}

The calcination temperature was based on our previous work [4]. For support the X-ray diffraction (XRD) measurements were taken. The calcination was carried out in flow of air for 180 min. The phase compositions of samples were determined by XRD method presented in Fig. 1. 


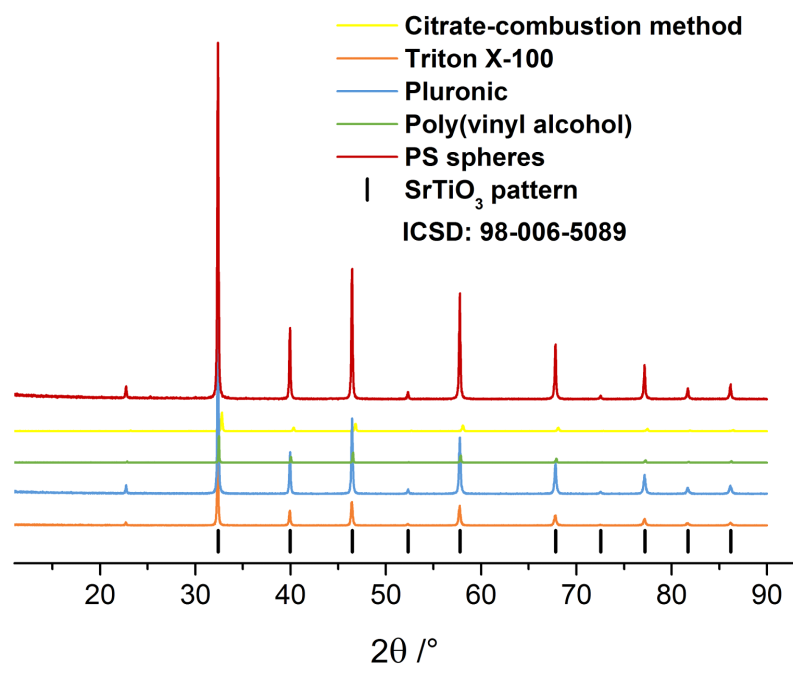

Fig. 1. X-ray diffraction pattern of $\mathrm{SrTiO}_{3}$ samples after calcination.

All of the obtained samples were single-phase materials $\left(\mathrm{SrTiO}_{3}\right)$. As expected the introduction of organic surfactants or soft templates prior calcination had no impact on $\mathrm{SrTiO}_{3}$ cubic structure (with the unit cell $a=3.9073 \pm 0.0005 \AA$. The synthesis procedure led to obtain materials that differ with microstructures as presented in Fig. 2.

The first three samples presented in Fig. 2. (A) $-(\mathrm{C})$ did not exhibit any regularity in their structure. Furthermore samples synthesized (presented in Fig. 2C and D) with the use of Pluronic P-123 appearance of macromesoporous structure was observed. Moreover, samples made with P-123 exhibit opal-like pores, that differ from pores of all other samples which are a consequence of crystallites or particles agglomeration. For samples with PS templates three-dimensionally ordered macroporous structure was observed. Still after sintering process only sample made with the use of P-123 sustained macromesoporous its native structure. The total porosity of pellets sintered at $1200^{\circ} \mathrm{C}$ with the use of Pluronic P-123 or Triton X-100 had the highest value - (57 \pm 3 vol. $\%)$, next group with lower value $(51 \pm 3$ vol.\%) was found for samples with only citric acid and with PS template. Lastly the lowest porosity was observed for samples when PVA was applied - $41 \pm 4$ vol. $\%$.
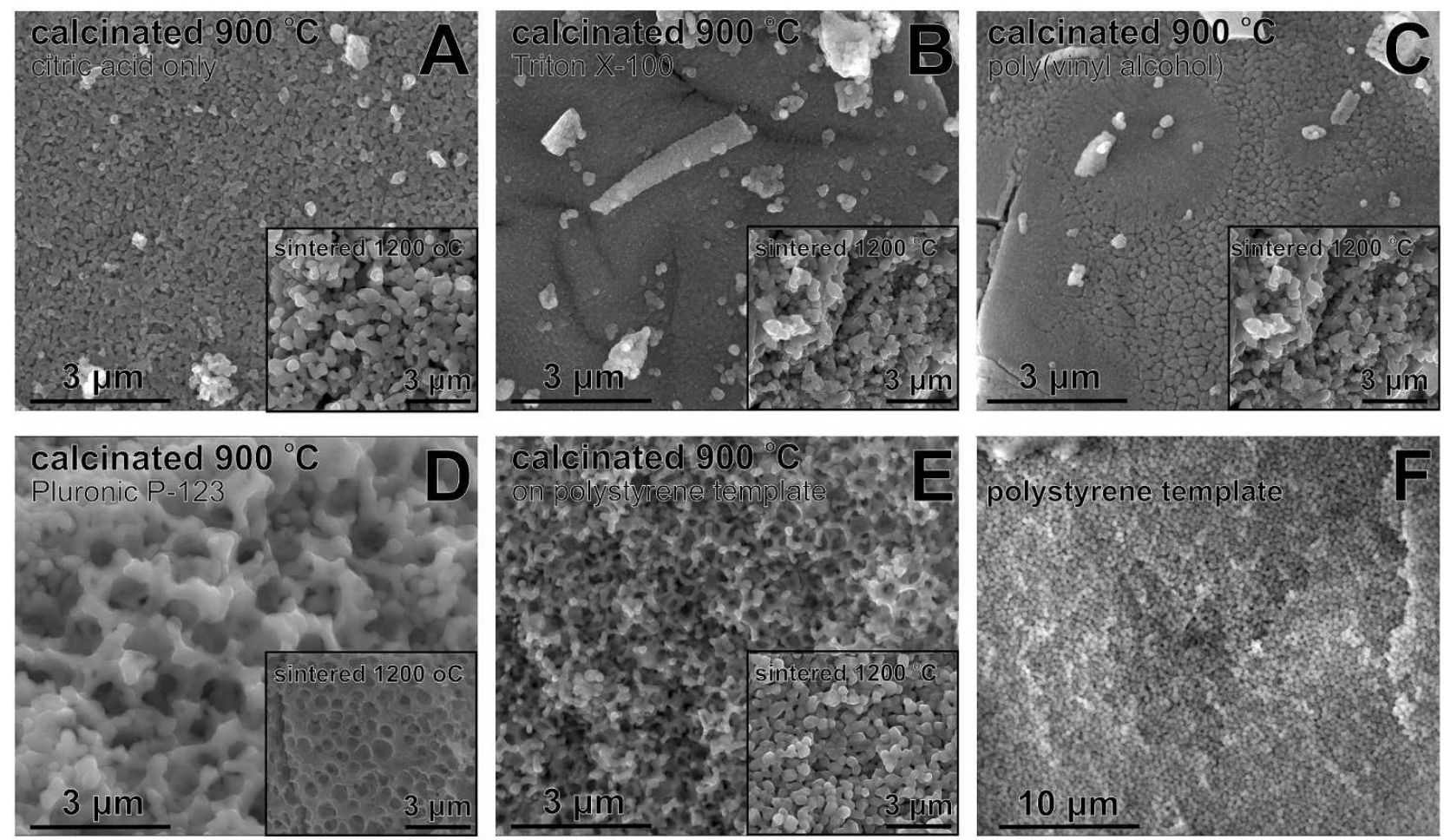

Fig. 2. The SEM microphotographs STO samples after calcination and sintering made with different surfactants: (A) only citric acid, (B) Triton X-100, (C) poly (vinyl alcohol), (D) Pluronic P-123, (E) soft polystyrene templates. The last picture $(\mathrm{F})$ shows polystyrene spheres used for sample E.

\section{Conclusions}

Strontium titanate $\left(\mathrm{SrTiO}_{3}\right)$ materials has been synthesized via sol-gel synthesis that differs by application of several kinds of pore former media. All approaches result in single-phase perovskite structure. However, the modification of synthesis path with surfactants and soft templates leads to significant differences in materials microstructure. As a consequence of surfactant usage, the random pores distribution was obtained in Pluronic P-123 modified samples. Additionally uniform 
pore structure was observed when polystyrene soft template was introduced during synthesis. However only P-123 modified STO samples have sustained their macromesoporous structure after sintering.

\section{Acknowledgments}

This work was financially supported by the National Science Center of the Republic of Poland, Grant No. 2014/14/E/ST5/00763.

\section{References}

[1] S. Sakka, J. Am. Chem. Soc. 127, 6135 (2005).

[2] S. Suthirakun, S.Ch. Ammal, G. Xiao, F. Chen, K. Huang, H.-C. Loye, A. Heyden, Solid State Ion. 228, 37 (2012)

[3] D. Grosso, C. Boissiere, L. Nicole, C. Sanchez, J. SolGel. Sci. Techn. 40, 141 (2006).

[4] E. Drożdż, Ł. Łańcucki, A. Łącz, J. Therm. Anal. Calorim. 125, 1225 (2016). 